\title{
Fine Structural Features and Electronic Structure of Core-Shell Structured Fe Nanoparticles Probed using TEM/STEM and EELS
}

\author{
C. M. Wang ${ }^{*}$, D. R. Baer ${ }^{*}$, M. H. Engelhard*, J. E. Amonette*, J.J. Antony ${ }^{* *}$, and Y. Qiang ${ }^{* *}$ \\ *Pacific Northwest National Laboratory, P.O. Box 999, Mail Stop K8-93, Richland, WA \\ 99352 \\ **Physics Department, University of Idaho, Moscow ID 83844-0903
}

Clean $\mathrm{Fe}^{0}$ exposed to air or an oxygen-including atmosphere will be nearly instantly oxidized [1] leading to formation of a surface oxide layer that slows or protects against further oxidation. Nanometer-sized iron particles exposed to oxygen-including environments show no exclusion from this rule. This leads to the Fe nanoparticles being covered by a thin layer of oxide to form core-shell structured Fe nanoparticles. The typical thickness of this oxide layer is $\sim 2-3 \mathrm{~nm}[2]$.

Even though a large body of literature exists in the field of Fe nanoparticles, no consistent understanding has yet emerged on the morphological structural features of the iron nanoparticles and their relationship to the native oxide skin. The structural nature of the oxide layer is unclear in many circumstances: is it $\mathrm{FeO}, \mathrm{Fe}_{2} \mathrm{O}_{3}$ (spinel and corundum), and $\mathrm{F}_{3} \mathrm{O}_{4}$, a combination or a different phase? Although small iron nanoparticles are generally round, once the size increases above $20 \mathrm{~nm}$ the particles often form a more crystalline shape. It is well established in high-temperature oxidation products, for example, that the phase and composition of the thick oxide layer formed on an Fe substrate depend on the distance of the layer from the innermost Fe-to-oxide interface such that a progression from $\mathrm{Fe}: \mathrm{FeO}: \mathrm{Fe}_{3} \mathrm{O}_{4}: \mathrm{Fe}_{2} \mathrm{O}_{3}$ occurs [3]. However, for the very thin oxide shell formed at room temperature on nanoparticles, it is hard to distinguish the spatial differences of the structure of the oxide shell as one moves from the external surface to the innermost Fe:Fe-oxide interface.

In this work, we report the fine structural features of core-shell structured Fe nanoparticles prepared by a room temperature (RT) sputter-gas-aggregation (SPA) process. The structural nature of the oxide shell on individual Fe nanoparticles was probed using electron energy loss spectroscopy (EELS) in a transmission electron microscope (TEM) and scanning TEM (STEM). This approach provides both structural features and the electronic structural information about the thin oxide shells on the $\mathrm{Fe}$ nanoparticles at a spatial resolution comparable to their size.

Core-shell nanostructured Fe particles formed by the SPA process and deposited at RT show morphologies of ranging from cube (defined by the $6\{100\}$ planes) to truncated rhombic dodecahedron (defined by the $6\{100\}$ planes and truncated by the $12\{110\}$ planes). No particles have been found with a morphology of regular rhombic dodecahedron (defined only by the $12\{110\}$ planes). HRTEM imaging and electron diffraction indicate that the oxide layer on the Fe surface is dominated by the $\mathrm{Fe}_{3} \mathrm{O}_{4} / \gamma$ $\mathrm{Fe}_{2} \mathrm{O}_{3}$ and maintains an invariable orientation relationship of $\mathrm{Fe}[001] / /$ oxide[110] and $\mathrm{Fe}(-200) / /$ oxide(004). 


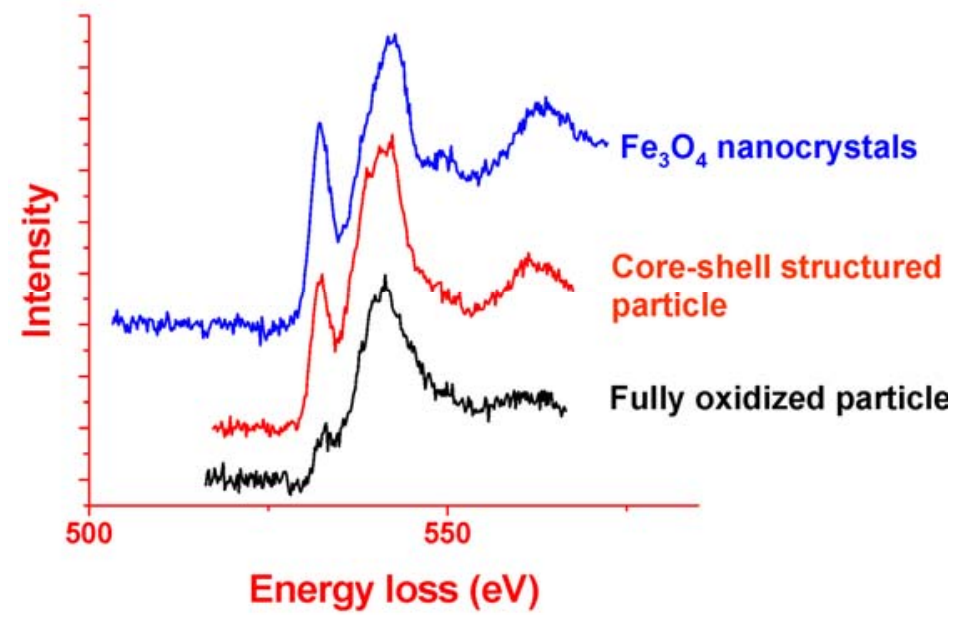

Fig. 1 Comparison of the EELS spectra collected on the core-shell Fe particles and $\mathrm{Fe}_{3} \mathrm{O}_{4}$ nanocrystal.

EELS has been used to probed into two types of representative particles: small particles that are fully oxidized and larger core-shell particles that possess an Fe core (Figure 1). We found that the $\mathrm{O} K$-edge spectra collected for the oxide shell in nanoparticles show distinct differences from those of known Fe oxides. Based on the results of spectral finger printing and quantum mechanical calculations, we conclude that the distances between the absorbing $\mathrm{O}$ and the next-nearest neighbor $\mathrm{O}$ atoms are more widely distributed than in standard $\mathrm{Fe}_{3} \mathrm{O}_{4}$ for both of these two types of particles. For small, fully oxidized particles, there is also a broadened distribution between the absorbing $\mathrm{O}$ and the nearest neighbor $\mathrm{O}$ atoms. These results clearly demonstrate that the coordination configuration in the oxide shell on Fe nanoparticles is defective compared to that of their bulk counterparts. Of the two types of particles examined in this work, the degree of disorder is greater for small, fully oxidized particles [2].

Acknowledgements: This work was supported by the U.S. Department of Energy (DOE) Office of Energy Research, Offices of Basic Energy Sciences and Biological and Environmental Research. Parts of the work were conducted in the William R. Wiley Environmental Molecular Sciences Laboratory (EMSL) a DOE User Facility operated by Battelle for the DOE Office of Biological and Environmental Research. Pacific Northwest National Laboratory is operated for the DOE under Contract DE-AC0676RLO 1830. At UI, the work is supported by DOE-AFCI project (DE-FC0708ID14926).

\section{References}

[1] N. Cabrera and N. F. Mott, Rep. Prog. Phys. 12, 163 (1947-1948).

[2] C. M. Wang, D. R. Baer, L. E. Thomas, J. E. Amonette, J. Antony, Y. Qiang, and G. Duscher, J. Appl. Phys. 98, 094308 (2005).

[3] E. Fromm, Kinetics of Metal-Gas Interactions at Low Temperature: Hydriding, Oxidation, Poisoning (Springer, Berlin, Heidelberg, New York, 1998). 